\title{
ON THE UBIQUITY OF HERRINGBONES IN FINITELY GENERATED LATTICES
}

\author{
I. RIVAL, W. RUCKELSHAUSEN AND B. SANDS
}

ABSTRACT. Every finitely generated infinite lattice of finite width contains a subset isomorphic to the "herringbone" or its dual.

1. Introduction. Aficionados of lattice diagrams have long noticed that a particular infinite lattice, which we illustrate in Figure 1, appears repeatedly in the diagrams of finitely generated infinite lattices. This lattice, the herringbone, can be seen within Rolf's diagrams of $F(2+2)$ and $F(1+4)$ [3]; more explicitly in Wille [4] and numerous other papers, usually as an integral part of a counterexample; in unpublished researches of the third author; finally in recent results of Bauer and Poguntke [1] and Poguntke and Sands [2], where it assumes a leading role.

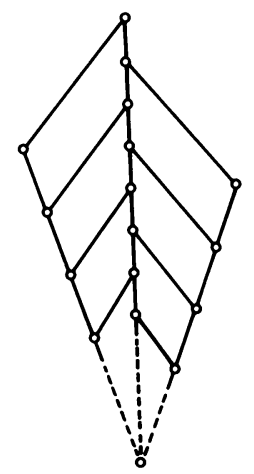

FIGURE 1. The herringbone

The main result of this paper may be considered as an explanation of the seemingly ubiquitous nature of the herringbone.

THEOREM. Every finitely generated infinite lattice of finite width contains a subset order-isomorphic to the herringbone or its dual.

If $L$ is a finitely generated infinite lattice of width three, Poguntke and Sands [2] have shown that $L$ will contain a sublattice isomorphic to the herringbone or its dual (see Figure 2(a) for an example). On the other hand, no such strengthening of our theorem is possible for lattices of width five (see Figure 2(b)).

Received by the editors November 6, 1979 and, in revised form, April 16, 1980.

AMS (MOS) subject classifications (1970). Primary 06B05. 


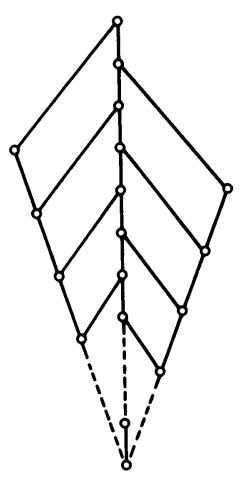

(a)

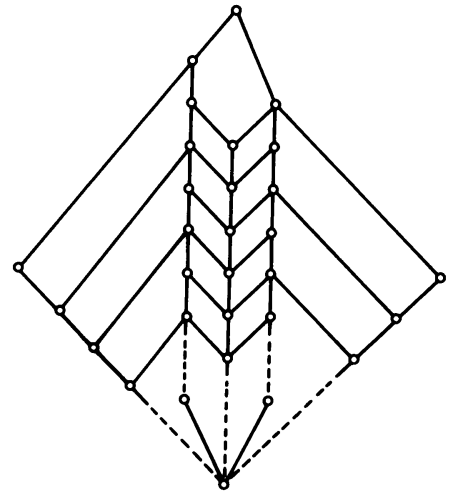

(b)

FIGURE 2

2. Infinite descending chains. Descending chains isomorphic to $\omega^{d}$ play an important role in this investigation. We first record, without proof, the following elementary observation.

LEMMA 1. Let $P$ be an ordered set of finite width, and let $C=\left\{c_{i} \mid i=1,2, \ldots\right\}$ and $D=\left\{d_{i} \mid i=1,2, \ldots\right\}$ be infinite subsets of $P$.

(a) If $c_{1}>c_{2}>c_{3}>\ldots$, and, for each $i, d_{i} \neq c_{i}$ but there exists $j>i$ such that $d_{i}>c_{j}$, then $C$ and $D$ contain subsequences $C^{\prime}=\left\{c_{i_{1}}>c_{i_{2}}>\ldots\right\}$ and $D^{\prime}=$ $\left\{d_{i_{1}}>d_{i_{2}}>\ldots\right\}$ respectively such that, for each $j, c_{i,} \nless d_{i_{j}}$ but $c_{i_{j+1}}<d_{i_{j}}$.

(b) If $d_{1}>d_{2}>d_{3}>\ldots$, and, for each $i, c_{i}<d_{i}$ but there exists $j>i$ such that $c_{i} \nless d_{j}$, then $C$ and $D$ contain subsequences $C^{\prime}=\left\{c_{i_{1}}>c_{i_{2}}>\ldots\right\}$ and $D^{\prime}=$ $\left\{d_{i_{1}}>d_{i_{2}}>\ldots\right\}$ respectively such that, for each $j, c_{i_{j}}<d_{i_{j}}$ but $c_{i_{j}} \nless d_{i_{j+1}}$.

The proof of the theorem rests in no little measure on the following simple, but important, result.

Proposition 2. Let $C=\left\{c_{1}>c_{2}>\ldots\right\}$ be a chain in a finitely generated lattice L. Then the subset $A_{C}=\left\{x \in L \mid\right.$ for all $\left.i, c_{i} \$ x\right\}$ is infinite. Moreover, for each $i$ there are $x_{i}, y_{i} \in A_{C}$ satisfying $x_{i} \vee y_{i} \notin A_{C}$ and $c_{i} \nless x_{i} \vee y_{i}$.

Proof. Let $L$ be a finitely generated lattice and let $C=\left\{c_{i} \mid i=1,2, \ldots\right\}$ be a subset of $L$ satisfying $c_{1}>c_{2}>\ldots$ Let us suppose that there is an $i$ such that, for all $x, y \in A_{C}$, either $c_{i} \leqslant x \vee y$ or $x \vee y \in A_{C}$. Then $A_{C} \cup\left[c_{i}\right)$, where $\left[c_{i}\right)=\{x \in$ $\left.L \mid c_{i}<x\right\}$, is a sublattice of $L$. Moreover, for all $j \geqslant i, A_{C} \cup\left[c_{j}\right)$ is a sublattice. As $R_{j}=L \backslash\left(A_{C} \cup\left[c_{j}\right)\right)$ is nonempty, it follows that $R_{j}$ must contain a generator $g_{j}$ of $L$, for each $j>i$. In particular, there is $j_{1}>i$ such that $g_{i}>c_{j_{1}}$. For the generator $g_{j_{1}} \in R_{j_{1}}, g_{j_{1}} \neq c_{j_{1}}$, whence $g_{i} \neq g_{j_{1}}$. Now there is $j_{2}>j_{1}$ such that $g_{j_{1}}>c_{j_{2}}$ and the generator $g_{j_{2}} \in R_{j_{2}}$ satisfies $g_{j_{2}} \ngtr c_{j_{2}}$. Therefore, the generators $g_{i}, g_{j_{1}}$, and $g_{j_{2}}$ are all distinct. In this way we construct an infinite set $\left\{g_{i}, g_{j_{1}}, g_{j_{2}}, \ldots\right\}$ of generators of $L$, which is a contradiction. We conclude from this that, for each $i$, there is $x_{i}$, $y_{i} \in A_{C}$ such that $x_{i} \vee y_{i} \notin A_{C}$ and $c_{i} \nless x_{i} \vee y_{i}$. 
Finally, that $A_{C}$ is infinite follows from Lemma 1. Indeed, from the conclusion above there are subsets $X=\left\{x_{i} \mid i=1,2, \ldots\right\}$ and $Y=\left\{y_{i} \mid i=1,2, \ldots\right\}$ of $A_{C}$, and a subsequence $c_{1}^{\prime}>c_{2}^{\prime}>\ldots$ of $C$ such that, for all $i, x_{i} \vee y_{i}>c_{i}^{\prime}$ but $x_{i} \vee y_{i} \ngtr c_{i-1}^{\prime}$. It follows that $\left\{x_{i} \vee y_{i} \mid i=1,2, \ldots\right\}$ and hence $X \cup Y$ is infinite, whence $A_{C}$ is infinite.

For an ordered set $P$, let $\mathcal{C}_{P}$ denote the set of all those subsets of $P$ which are isomorphic to $\omega^{d}$. In the case that $P$ is a lattice of finite width, we can endow $\mathcal{C}_{P}$ with a strict order.

Let $C, D \in \mathcal{C}_{P}$. We write $C<D$ if, for any infinite subset $D^{\prime}$ of $D$, there is an infinite subset $C^{\prime}$ of $C$ such that

(C1) For every $c \in C^{\prime}$ and for every $d \in D^{\prime}, c \ngtr d$, and

(C2) there is a map $f$ of $C^{\prime}$ to $D^{\prime}$ satisfying: $c<c^{\prime}$ if and only if $c<f\left(c^{\prime}\right)$.

REMARKs. 1. An instance of this ordering is illustrated in Figure 3.

2. If $C<D$ in $\mathcal{C}_{P}$, and $C^{\prime} \subseteq C, D^{\prime} \subseteq D$, and $f: C^{\prime} \rightarrow D^{\prime}$ are as above, then $c<c^{\prime}$ in $C^{\prime}$ if and only if $f(c)<f\left(c^{\prime}\right)$ in $D^{\prime}$; that is, $f$ is strict order-preserving.

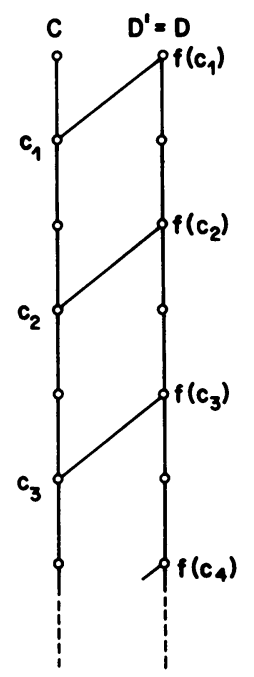

Figure 3

LEMMA 3. Let $L$ be a lattice of finite width. Then $<$ is a strict order on $\mathcal{C}_{L}$.

Proof. Let $C, D \in \mathcal{C}_{L}$. If $D<C$ then there is an infinite subset $D^{\prime}$ of $D$ such that for each $d \in D^{\prime}$ and for each $c \in C, d \neq c$. If also $C<D$ then there is an infinite subset $C^{\prime}$ of $C$ and a map $f$ of $C^{\prime}$ to $D^{\prime}$ satisfying $c<(c)$ for each $c \in C^{\prime}$. As $f(c) \in D^{\prime}$ this is a contradiction. Therefore, $<$ is antisymmetric.

Let $L$ have width $n$. We now show that $<$ is transitive on $\bigodot_{L}$. To this end let $C$, $D$, and $E$ be members of $C_{L}$ satisfying $C<D$ and $D<E$. Let $E^{\prime}$ be an infinite subset of $E$. Then there is an infinite subset $D^{\prime}$ of $D$ such that

(i) for each $d \in D^{\prime}$ and each $e \in E^{\prime}, d \neq e$, and

(ii) there is a map $g$ of $D^{\prime}$ to $E^{\prime}$ satisfying: $d<d^{\prime}$ if and only if $d<g\left(d^{\prime}\right)$. 
Likewise, there is an infinite subset $C^{\prime}$ of $C$ such that

(i) for each $c \in C^{\prime}$ and each $d \in D^{\prime}, c \neq d$, and

(ii) there is a map $f$ of $C^{\prime}$ to $D^{\prime}$ satisfying: $c<c^{\prime}$ if and only if $c<f\left(c^{\prime}\right)$.

Let $C^{\prime}=\left\{c_{1}>c_{2}>\ldots\right\}$. Let us suppose that, for some $i, c_{i}<g\left(f\left(c_{2 n+i}\right)\right)$. We shall show that $\left\{c_{i+j} \vee f\left(c_{2 n+i-j}\right) \mid j=0,1,2, \ldots, n\right\}$ is an $(n+1)$-element antichain in $L$, which is impossible since $L$ has width $n$. Note that, for each $j=$ $0,1,2, \ldots, n$,

$$
c_{i+j} \leqslant c_{i}<g\left(f\left(c_{2 n+i}\right)\right)<g\left(f\left(c_{2 n+i-j}\right)\right) .
$$

Let $0 \leqslant k<k^{\prime} \leqslant n$, and set $c=c_{2 n+i-k}$ and $c^{\prime}=c_{2 n+i-k^{\prime}}$. Observe that $c<c^{\prime}$. Then $c_{i+k} \vee f(c)<g(f(c))$. It follows that $f\left(c^{\prime}\right) \nless c_{i+k} \vee f(c)$, for otherwise $f\left(c^{\prime}\right)<$ $g(f(c))$ and so $f\left(c^{\prime}\right) \leqslant f(c)$ which is a contradiction to $c<c^{\prime}$. Therefore,

$$
c_{i+k^{\prime}} \vee f\left(c^{\prime}\right) \$ c_{i+k} \vee f(c)
$$

Since $k^{\prime}<n, c^{\prime}=c_{2 n+i-k^{\prime}} \leqslant c_{i+k^{\prime}}$, so $c_{i+k^{\prime}} \bigvee f\left(c^{\prime}\right)<f\left(c_{i+k^{\prime}}\right)$. Thus, $c_{i+k} \nless c_{i+k^{\prime}} \bigvee$ $f\left(c^{\prime}\right)$ and

$$
c_{i+k} \vee f(c) \$ c_{i+k^{\prime}} \vee f\left(c^{\prime}\right)
$$

Therefore, $c_{i+k} \vee f(c)$ is noncomparable to $c_{i+k^{\prime}} \vee f\left(c^{\prime}\right)$ for all $0<k<k^{\prime}<n$, which is impossible. We conclude that, for all $i$,

$$
c_{i} \nless g\left(f\left(c_{2 n+i}\right)\right) \text {. }
$$

Finally, let $C^{\prime \prime}=\left\{c_{2 n k+1} \mid k=0,1,2, \ldots\right\}$. For every $c \in C^{\prime \prime}$ and for every $e \in E^{\prime}, c \ngtr e$ (for otherwise, $f(c)>e$ for some $c \in C^{\prime \prime}$ and some $e \in E^{\prime}$, which is impossible since $D<E$ ). Now, let $h=\left.g \circ f\right|_{c^{\prime \prime}}$. If $c_{2 n k+1}<c_{2 n k^{\prime}+1}$ then

$$
c_{2 n k+1}<f\left(c_{2 n k^{\prime}+1}\right)<g\left(f\left(c_{2 n k^{\prime}+1}\right)\right)=h\left(c_{2 n k^{\prime}+1}\right) .
$$

If $c_{2 n k+1}<h\left(c_{2 n k^{\prime}+1}\right)$ and $c_{2 n k^{\prime}+1}<c_{2 n k+1}$ then $k^{\prime}>1+k$, so $c_{2 n k^{\prime}+1}<c_{2 n+2 n k+1}$ and $c_{2 n k+1}<h\left(c_{2 n+2 n k+1}\right)$ which contradicts (*) with $i=2 n k+1$. This completes the proof.

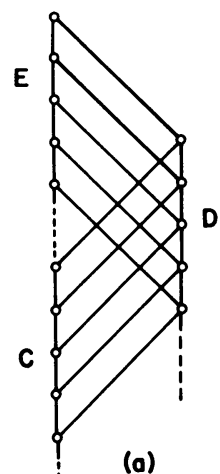

(a)

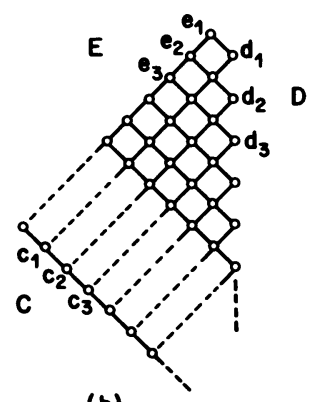

(b)

\section{FIGURE 4}

If $\boldsymbol{P}$ is not a lattice then the relation $<$ on $\mathcal{C}_{\boldsymbol{P}}$ need not be transitive, even if $\boldsymbol{P}$ has width two (see Figure 4(a)). In fact, it is not hard to show that, for an ordered set $P,<$ is a strict order on $\mathcal{C}_{P}$ if and only if $P$ contains no subset isomorphic to the 
ordered set of Figure 4(a). If $P$ is a lattice with arbitrarily large finite antichains, then again $<$ on $\mathcal{C}_{P}$ need not be transitive (see Figure $4(\mathrm{~b})$ ).

LEMMA 4. Let $P$ be an ordered set, let $C, D \in \mathcal{C}_{P}$, and let $C^{\prime}, D^{\prime}$ be infinite subsets of $C, D$, respectively. If $C<D$ then $C^{\prime}<D^{\prime}$.

LEMMA 5. Let $L$ be a lattice of finite width. Then $\mathcal{C}_{L}$ has finite length.

Proof. We show by induction on $n$ that, for any chain $C_{1}<C_{2}<\cdots<C_{n}$ of members of $C_{L}$ and for any selection $x_{i} \in C_{i}, i=1,2, \ldots, n$, there is a system $a_{1}$, $a_{2}, \ldots, a_{n}$ of distinct representatives of $C_{1}, C_{2}, \ldots, C_{n}$ such that $a_{i}<x_{i}$ for each $i=1,2, \ldots, n$ and $\left\{a_{1}, a_{2}, \ldots, a_{n}\right\}$ is an $n$-element antichain. As $C_{i}<C_{n}$ for each $i=1,2, \ldots, n-1$, there exist chains $C_{i}^{\prime}$ and maps $f_{i}$ of $C_{i}^{\prime}$ to $C_{n}$ satisfying (C1) and (C2). From Lemma $4 C_{1}^{\prime}<C_{2}^{\prime}<\ldots$ For each $i=1,2, \ldots, n-1$, set $x_{i}^{\prime}=\inf \left\{x_{i}, \sup C_{i}^{\prime}\right\}$. According to the induction hypothesis, there is, for each $i=1,2, \ldots, n-1$, an element $a_{i} \in C_{i}^{\prime}$ such that $a_{i}<x_{i}^{\prime}$ and such that $\left\{a_{1}, a_{2}, \ldots, a_{n-1}\right\}$ is an $(n-1)$-element antichain. Finally, let $a_{n}$ be an element of $C_{n}$ satisfying $a_{n}<\inf \left\{f_{i}\left(a_{i}\right) \mid i=1,2, \ldots, n-1\right\}$. Then $\left\{a_{1}, a_{2}, \ldots, a_{n}\right\}$ is an $n-$ element antichain. This shows that $\mathcal{C}_{L}$ has length at most $n-1$ if $L$ has width n.

$e_{L}$ may have infinite chains even if $L$ contains no infinite antichains (see Figure 5).

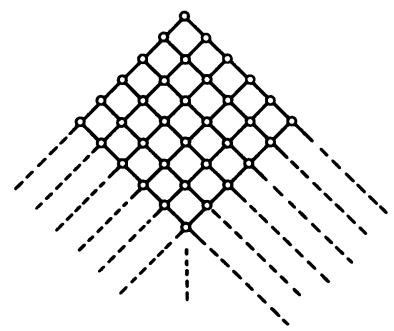

Figure 5

Notice that, if $L$ is an infinite lattice of finite width, Lemma 5 guarantees that $\mathcal{C}_{L}$ will contain minimal elements.

LeMma 6. Let $C$ be a minimal element of $\mathcal{C}_{L}$ and let $D$ be an infinite subset of $C$. Then $D$ is also a minimal element of $\mathcal{C}_{L}$ (although noncomparable with $C$ ).

Proof. By way of contradiction, assume that $E \in \mathcal{C}_{L}$ is such that $E<D$. We shall prove that $E<C$, which is impossible. Let $E^{\prime} \subseteq E$ and $f: E^{\prime} \rightarrow D$ be as in the definition of $<$. Let $C^{\prime}$ be an infinite subset of $C$, define

$$
c_{1}=\max C^{\prime}, \quad e_{1}=\max \left\{e \in E^{\prime} \mid f(e)<c_{1}\right\},
$$

and inductively define, for each $i>1$,

$$
c_{i}=\max \left\{c \in C^{\prime} \mid c \neq e_{i-1}\right\}, \quad e_{i}=\max \left\{e \in E^{\prime} \mid f(e)<c_{i}\right\} .
$$

(These definitions are possible since $C^{\prime}$ and $E^{\prime}$ are isomorphic to $\omega^{d}$.) It is now easy to see that the set $E^{\prime \prime}=\left\{e_{1}, e_{2}, \ldots\right\}$ and the map $g: E^{\prime \prime} \rightarrow C^{\prime}$ defined by $g\left(e_{i}\right)=c_{i}$ for each $i$, demonstrate that $E<C$. 
3. Proof of the theorem. Let $L$ be an infinite, finitely generated lattice of finite width. We may suppose that $e_{L} \neq \varnothing$. By Lemma 5 , we may choose a minimal member $C=\left\{c_{1}>c_{2}>c_{3}>\ldots\right\}$ of $\mathcal{C}_{L}$. In view of Proposition 2, for each $c_{i} \in C$ there are elements $x_{i}, y_{i}$ in $A_{C}$ such that $z_{i}=x_{i} \vee y_{i} \ngtr c_{i}$ and $z_{i} \notin A_{C}$; that is, $z_{i}>c_{j}$ for some $j>i$. By Lemma 1 , there is an infinite subset $C^{\prime}=\left\{c_{i_{1}}>c_{i_{2}}\right.$ $>\ldots\}$ of $C$ and a corresponding infinite chain $Z=\left\{z_{i_{1}}>z_{i_{2}}>\ldots\right\}$ such that, for each $j, z_{i j} \ngtr c_{i j}$ and $z_{i j} \geqslant c_{i j+1}$. From Lemma 6, $C^{\prime}$ is minimal in $\mathcal{C}_{L}$; observing also that $A_{C}=A_{C^{\prime}}$, we see that $C^{\prime}$ may be taken to be $C$, that is, we may assume that $Z=\left\{z_{1}>z_{2}>\ldots\right\}$ and $z_{i} \ngtr c_{i}, z_{i}>c_{i+1}$ for each $i$. (This tactic of identifying an infinite set with an infinite subset possessing certain desirable properties we call, naturally enough, relabeling.) Next, since $z_{i+1}<z_{i}=x_{i} \vee y_{i}$, we may assume that, for each $i, x_{i} \$ z_{i+1}$ and so $x_{i} \$ c_{i+2}$. An application of Lemma 1 followed by a relabeling yields $x_{1}>x_{2}>\ldots$ Let $X=\left\{x_{1}, x_{2}, \ldots\right\}$. Recall that $x_{i} \in A_{C}$, and thus for all $j, x_{i} \neq c_{j}$. It follows that, if for each $c_{i}$ there were $x_{j}$ such that $x_{j}<c_{i}$, we would have $X<C$, a contradiction; thus there exists $k$ such that, for all $n>k$, $x_{n} \$ c_{k}$. By relabeling, we may assume that for all $n, x_{n} \$ c_{1}$. It is now evident that the subset $C \cup Z \cup X$ is isomorphic to the ordered set illustrated in Figure 6 . Selecting the subset consisting of $\left\{c_{i} \mid i\right.$ even $\} \cup Z \cup\left\{x_{i} \mid i\right.$ even $\}$ produces the required herringbone.

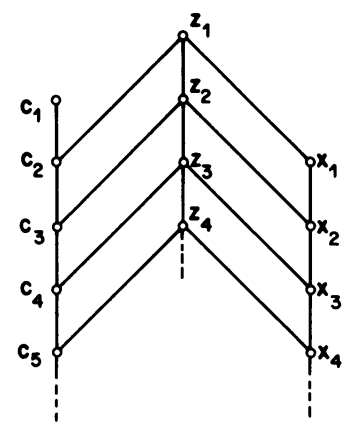

FIGURE 6

\section{REFERENCES}

1. H. Bauer and W. Poguntke, Lattices of width three: an example and a theorem, Contributions of General Algebra, (Proc. Klagenfurt Conf., 1978), Verlag Johannes Heyn, Klagenfurt, 1979, pp. 47-54.

2. W. Poguntke and B. Sands, On finitely generated lattices of finite width, Canad. J. Math. (to appear).

3. H. L. Rolf, The free lattice generated by a set of chains, Pacific J. Math. 8 (1958), 585-595.

4. R. Wille, Jeder endlich erzeugte, modulare Verband endlicher Weite ist endlich, Mat. Casopis Sloven. Akad. Vied. 24 (1974), 77-80.

Department of Mathematics \& Statistics, University of Calgary, Calgary T2N 1N4, Alberta, Canada 